\title{
DIELECTRIC AND OPTICAL STUDIES OF HOMOLOGOUS SERIES OF BICYCLO $[2,2,2]$ OCTANE DERIVATIVES WITH -NCS TERMINAL GROUP
}

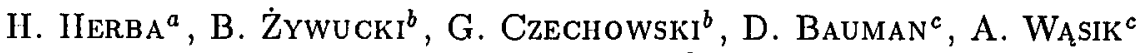 \\ AND J. JADŻYN ${ }^{b}$ \\ ${ }^{a}$ Department of Physics, University of Technology, W. Pola 2, 35-969 Rzeszów, Poland \\ ${ }^{b}$ Institute of Molecular Physics, Polish Academy of Sciences \\ Smoluchowskiego 17, 60-179 Poznan, Poland \\ cInstitute of Physics, Poznań University of Technology \\ Piotrowo 3, 60-965 Poznań, Poland
}

(Received January 24, 1995)

\begin{abstract}
The static dielectric constants and the optical birefringence for four members of the homologous series of bicyclo[2,2,2]octane derivatives with -NCS terminal group (pentyl to octyl) have been measured as a function of temperature. From the dielectric anisotropy, the optical birefringence as well as the polarized absorption spectra of the dichroic dye dissolved in the liquid crystalline host the order parameter has been determined. The results obtained by using various experimental methods have been compared with those calculated from the Maier-Saupe mean field theory. It has been found that the order parameter for the successive homologues shows alternation similar to that exhibited for the nematic-isotropic transition temperature.
\end{abstract}

PACS numbers: $61.30 . \mathrm{Gd}, 77.22 . \mathrm{Ch}, 78.20 . \mathrm{Fm}$

\section{Introduction}

In recent years a rapid progress of the liquid crystal displays (LCDs) technology has been observed. Among the various possible display technologies, LCDs fascinate because of their low power consumption, design flexibility and flatness, viewability in bright light and low costs. The use of LCDs was initially restricted to simple applications, such as wrist-watches, pocket calculators and measuring instruments, where information displayed is rather limited. Today, the invention of new electro-optical effects offers LCDs with high information contents, which can 
be applied in automotive instruments panels, computer and TV screens, telecommunication and in the important area of office automation. Such remarkable development in the LCD technology is closely connected with the design and production of novel liquid crystal materials with specific physical properties. The electro-optical parameters of LCD, such as threshold voltage, response times, contrast ratio and multiplexing capability are strongly dependent on the anisotropy in optical, electrical, magnetic and elastic properties of liquid crystal materials. In the nematic liquid crystalline phase the anisotropy of these properties arises because of the presence of the long-range orientational order, which is the most fundamental characteristics of this mesophase. Therefore, it is not astonishing that since early days of describing the nematic phase by molecular theories [1,2] intensive scientific research is directed to the investigation of the degree of order.

In this paper we have studied the dielectric and optical properties of four members (pentyl to octyl) of homologous series of bicyclo[2,2,2]octane derivatives with -NCS terminal group. Because of their low viscosity, low melting enthalpies and the existence of the nematic phase only, these substances can be highly useful as components of the technologically important liquid crystal mixtures $[3,4]$. In our investigations we have measured the dielectric constants and the optical birefringence of the liquid crystal as well as the polarized absorption spectra of the dichroic dye dissolved in the liquid crystalline host as a function of temperature. From the dielectric and optical data we have evaluated the order parameter, which describes the orientational order in the uniaxial phases of liquid crystals.

\section{Experimental}

\subsection{Malerials}

The molecular structure of nematogenic substances studied in this paper together with their crystal-nematic $\left(T_{\mathrm{CN}}\right)$ and nematic-isotropic $\left(T_{\mathrm{NI}}\right)$ phase transition temperatures are gathered in Table I. The substances were kindly supplied by Prof. R. Dąbrowski from the Military Academy of Technology in Warsaw and were used in the investigations without further purification. 4-dimethylamino-4'-nitrostilben (DANS), which was taken as a guest probe in the absorption measurement, was synthesized and chromatographically purified in the Institute of Dyes at Eódź Technical University. It was dissolved in the liquid crystalline hosts at a concentration of $5 \times 10^{-3} \mathrm{M}$.

\subsection{Apparalus}

The determination of the liquid crystal birefringence, $\Delta n$, was made on the basis of Newton rings observed at $\lambda=546 \mathrm{~nm}$ by means of the polarizing microscope (ZPO, Warsaw) equipped with the heating stage. The shift of the distance between interference rings by the sample heating was used to determine the values of $\Delta n$ as a function of temperature. The planar alignment of the molecules between flat glass surface and glass lens was obtained using rubbing procedure. The temperature was stabilized with the accuracy of $\pm 0.05 \mathrm{~K}$. The error in the estimation of $\Delta n$ was about $1 \%$. The further details of the method are described elsewhere [5]. 
TABLE I

Molecular structure and nematic phase region for liquid crystals investigated.

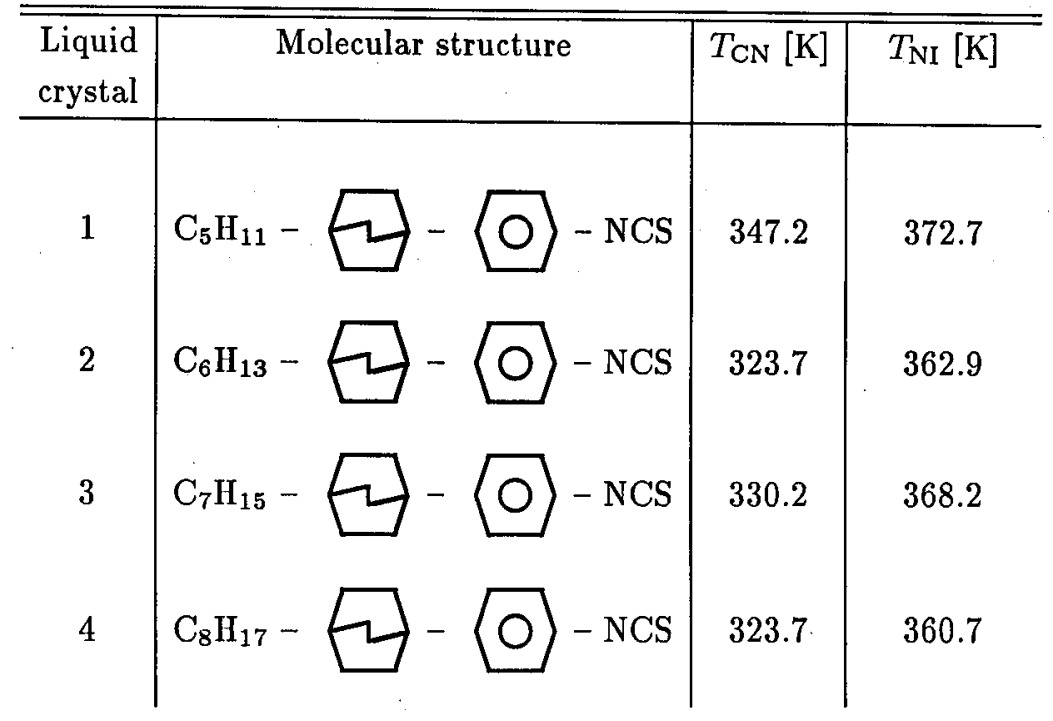

The static electric permittivity versus temperature was measured at the frequency of $10 \mathrm{kHz}$ using a Wayne-Kerr 6425 bridge. In order to obtain the values of the permittivity components of the oriented liquid crystals, the condenser was placed in an electromagnet $(B=0.6 \mathrm{~T})$ first in a position, where the magnetic field was parallel to the measuring electric field $\boldsymbol{E}\left(\varepsilon_{\|}\right)$and then rotated such that $\boldsymbol{E}$ and $\boldsymbol{B}$ were orthogonal $\left(\varepsilon_{\perp}\right)$. It was estimated that the overall uncertainty in $\boldsymbol{\varepsilon}$ was about $0.1 \%$. The accuracy of the temperature stabilization was $\pm 0.01 \mathrm{~K}$.

The polarized absorption spectra of the dye dissolved in liquid crystals were recorded using a SPECORD M-40 spectrophotometer (Carl Zeiss Jena) equipped with UV polarizers. The measurements were made as a function of the temperature in "sandwich" cells of $20 \mu \mathrm{m}$ in thickness. The homogeneous planar orientation of the guest and host molecules was achieved by means of treatment of the glass surfaces of the cells with polyimide and by rubbing process additionally. The temperature of the cells was regulated and controlled with the accuracy of $\pm 0.1 \mathrm{~K}$.

\section{Determination of order parameter}

For the most purposes the long-range orientational order can be described with sufficient accuracy by the second-rank order parameter, which is equivalent to that introduced by Saupe [6] and is defined as follows:

$$
S=\frac{1}{2}\left(3\left\langle\cos ^{2} \beta\right\rangle-1\right),
$$

where $\beta$ is the angle between the long molecular axis and the direction of the orientation and brackets denote an average over all possible molecules orientations. 
The most experimental methods used for determination of the order parameter in the liquid crystals require the sample to be macroscopically oriented. Such an orientation can be produced by using the electric or magnetic fields, surface forces or shear stress. If the macroscopic molecular alignment is complete it is possible to obtain information about the order parameter from measurements of the anisotropy of any bulk physical quantity such as for example electric permittivity, $\Delta \varepsilon$, refractive index, $\Delta n$, and diamagnetic susceptibility, $\Delta \chi$ [7]. The magnetic susceptibility is preferred parameter to the determination of $S$ because the diamagnetic moments of the molecules are very small, and consequently the interactions between these moments can be ignored. As a result, the field acting on a molecule can be taken to be equal to the externally applied magnetic field. Assuming that the liquid crystal molecules are axially symmetric due to the effectively free rotation around the long molecular axis, Zvetkoff [8] suggested many years ago that $\Delta \chi$ is a direct measure of the nematic order parameter $S$ and he defined $S$ as follows [7-9]:

$$
S=\frac{\Delta \chi}{\Delta \chi_{\max }}
$$

where $\Delta \chi_{\max }$ denotes the anisotropy of the susceptibility in the perfectly aligned phase.

Other as in the case of the magnetic field, the effective electric field "seen" by one molecule is a superposition of the external field, plus the field due to all other dipoles. Therefore the direct relation between $S$ and $\Delta \varepsilon$ or $\Delta n$ is not so evident as in the case of $\Delta \chi$. On the other hand, the anisotropy of the dielectric constant and the optical birefringence are very conveniently measurable quantities, which depend on temperature in the similar way (mainly $\Delta n$ ) as the order parameter $S$. Therefore, we assume $S$ to be directly proportional to $\Delta \varepsilon$ and $\Delta n$ [5]. As a result we have determined the order parameter from the following relations:

and

$$
S=\frac{\Delta \varepsilon}{\Delta \varepsilon_{\max }}
$$

$$
S=\frac{\Delta n}{\Delta n_{\max }}
$$

where $\Delta \varepsilon_{\max }$ and $\Delta n_{\max }$ are, respectively, the dielectric anisotropy and the optical birefringence for (hypothetical) completely ordered liquid crystal.

$\Delta \varepsilon_{\max }$ and $\Delta n_{\max }$ should be obtained in principle from the measurements of the electric permittivity and the refractive index in the solid state. Another possibility is application of the extrapolation procedure $[5,10,11]$. Assuming that $S$ changes with the temperature approximately as follows:

$$
S=\left(1-T / T_{\mathrm{NI}}\right)^{\gamma}
$$

and is equal to 1 at $0 \mathrm{~K}$, we are able to obtain $\Delta \varepsilon_{\max }$ and $\Delta n_{\max }$ plotting $\log \Delta \varepsilon$ or $\log \Delta n$ against $\log \left(1-T / T_{\mathrm{NI}}\right)$ and using extrapolation to the absolute zero.

Previously [5] it was shown for the well known liquid crystal PAA that the values of $S$ obtained from the dielectric and optical birefringence data using the method described above are in very good agreement with the data described in literature and determined by other methods ( $\Delta \chi$, NMR, IR). 
Another simple way to obtain information about the long-range orientational order in nematic phase is utilization of the liquid crystal as a host matrix for a dichroic dye probe ("guest-host" effect $[12,13]$ ). If the molecular structure of the dye is similar in size and shape to that of the liquid crystal molecules, the dye adapts the same anisotropic orientation as the nematic host phase. Knowing the direction of the transition moment with respect to the principal axis of the dye molecule, the polarized absorption spectra can give qualitative information about the molecular orientation, which is a very often used method. Then the order parameter $S$ can be easily obtained from the formula $[14,15]$

$$
S=\frac{A_{\|}-A_{\perp}}{A_{\|}+2 A_{\perp}} \frac{1}{1-\frac{3}{2} \sin ^{2} \theta}
$$

where $A_{\|}$and $A_{\perp}$ denote the absorbances at $\lambda_{\max }$ for the dye in the host medium when the electric vector of the incident light is, respectively, parallel and perpendicular to the director. $\theta$ is the angle between the absorption transition moment direction and the long axis of the dye molecule.

If $\theta=0^{\circ}$, Eq. (6) reduces to the equation given by White and Taylor [16]

$$
S=\frac{A_{\|}-A_{\perp}}{A_{\|}+2 A_{\perp}} \text {. }
$$

In our experiment we have chosen the stilbene derivative dye DANS whose molecular length is comparable to that of the liquid crystals used. This dye added to the nematic hosts at a very low concentration $\left(5 \times 10^{-3} \mathrm{M}\right)$ used in this study does not change the nematic-isotropic phase transition temperature within experimental uncertainty, which indicates that it does not perturb the arrangement of the liquid crystal molecules [17]. It seems that in the case of the dye probe DANS the assumption that the orientation of the guest reflects the orientation of the matrix is well satisfied. The longest wavelength absorption transition moment of DANS molecule is almost parallel to the long axis of the molecule $[18,19]$, therefore we can assume the angle $\theta$ to be $0^{\circ}$.

\section{Results}

Figure 1 shows the principal electric permittivities in the nematic $\left(\varepsilon_{\|}\right.$and $\left.\varepsilon_{\perp}\right)$ and isotropic $\left(\varepsilon_{\text {iso }}\right)$ phases for four members of the homologous series of the liquid crystal investigated as a function of temperature. The average values of the dielectric constant $\bar{\varepsilon}=\left(\varepsilon_{\|}+2 \varepsilon_{\perp}\right) / 3$ in the nematic phase, calculated from the measured $\varepsilon_{\|}$and $\varepsilon_{\perp}$ are also given in the figure. The dielectric anisotropy, $\Delta \varepsilon$ and the optical birefringence, $\Delta n$ versus reduced temperature $T^{*}=T / T_{\mathrm{NI}}$ for all the members of the series are presented in Figs. 2 and 3, respectively. Figure 4 shows the temperature dependence of the order parameter $S$ determined from the measurements of the anisotropy of the dielectric constant (squares) and the refractive index (circles) as well as from the polarized absorption spectra of DANS (triangles) for all the liquid crystals investigated. The solid line in these figure represents the results of the Maier-Saupe mean field theory of nematics [1]. Figure 5 presents the values of the order parameter, $S$, estimated from the absorption measurements at three reduced temperatures as a function of the alkyl chain length of the liquid crystal studied ( $n$ - number of carbon in the chain). 


\section{Discussion}

From Fig. 1 it is seen that the dielectric anisotropy of the liquid crystals investigated is positive which is due to the strong permanent electric dipole moments of the -NCS group parallel to the long axes of the molecules. The values of $\Delta \varepsilon$ of the series member with a given $n$ are similar to those of the corresponding member of $n$ CHBT's [20] and lower than $\Delta \varepsilon$ of the member of $n$ CB's [21] and PCIIn's [22]. Similarly as in the case of the all above-mentioned series of liquid crystals, the values of $\bar{\varepsilon}$ in the vicinity of the nematic-isotropic phase transition temperature are for the compounds studied slightly lower than the values of $\varepsilon_{\text {iso }}$. This difference is attributed to the antiparallel alignment of the molecules with the strong dipole moment in the nematic phase [23, 24].

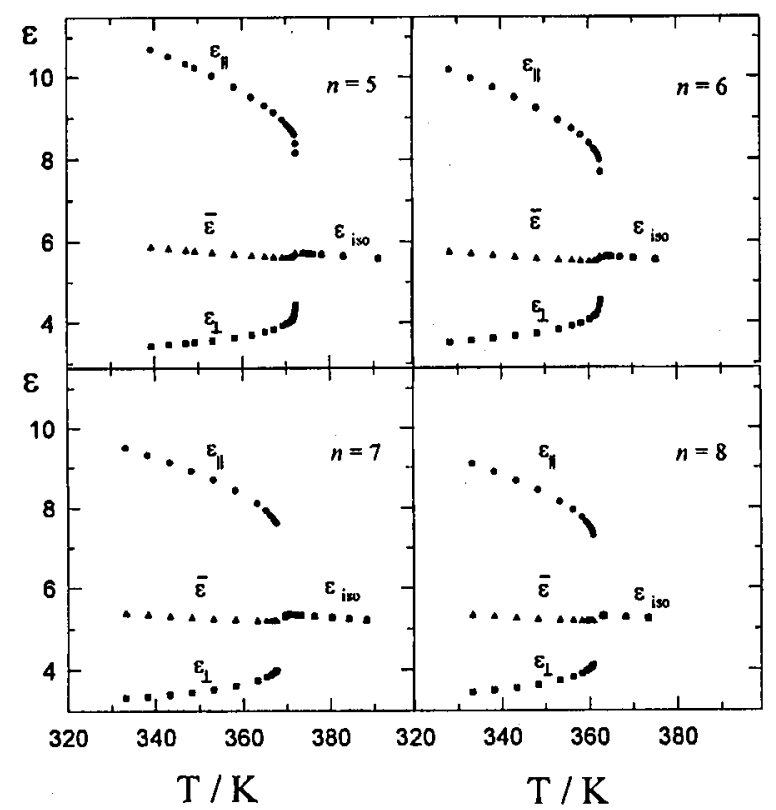

Fig. 1. Dielectric constants of liquid crystals investigated versus temperature.

Figures 2 and 3 indicate that both the dielectric anisotropy and the optical birefringence of the liquid crystals investigated decrease with the rise of temperature and jump to zero at the clearing point. The temperature dependence of $\Delta \varepsilon$ and $\Delta n$ resembles that of the order parameter $S$, presented in Fig. 4. From this figure it is seen that the character of the changes of $S$ with the increase in the temperature is similar for all the liquid crystals investigated and is in fairly good agreement with the Maier-Saupe theory. However, the values of $S$ at the same reduced temperature are different for various liquid crystals, which indicates the influence of the alkyl chain length on the long-range orientational order. The characteristic odd-even effect [25], explained as the alternation of interaction between alkyl chains [26], is observed; the compounds with the odd number of carbon in 

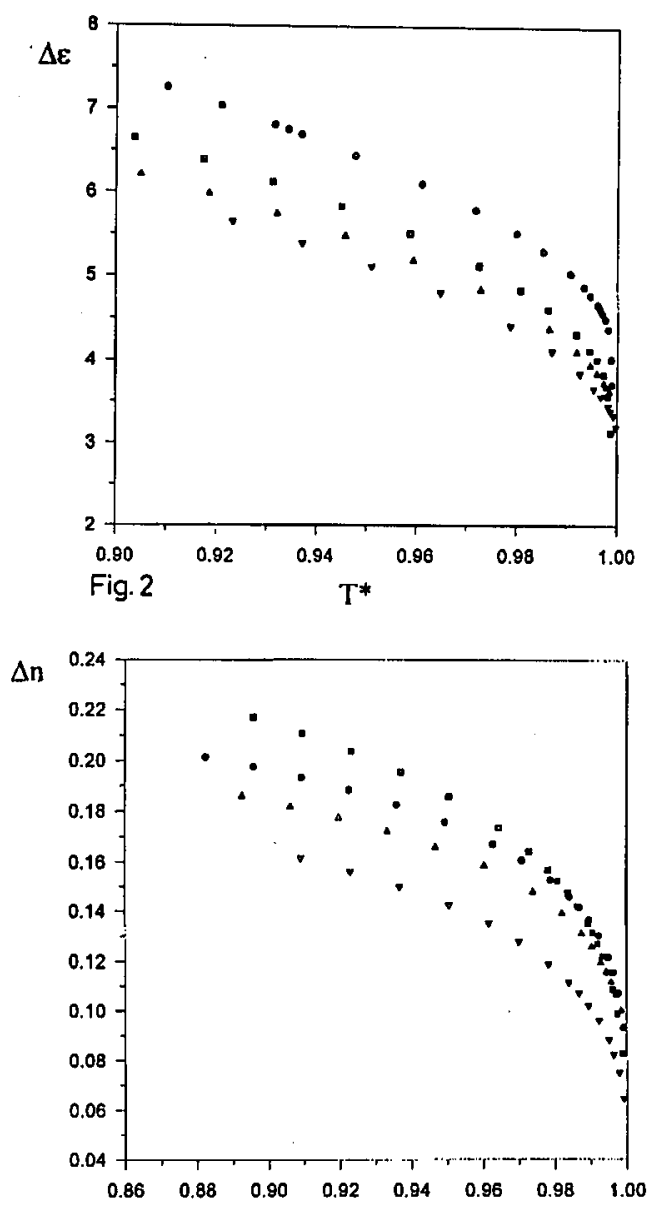

Fig.3

$\mathrm{T}^{*}$

Fig. 2. Dielectric anisotropy of liquid crystals investigated as a function of reduced temperature: $\bullet-n=5, \mathbf{\square}-n=6, \Delta-n=7, \nabla-n=8$.

Fig. 3. Optical birefringence of liquid crystals investigated as a function of reduced temperature: $\bullet-n=5, \mathbf{n}-n=6, \Delta-n=7, \nabla-n=8$.

the alkyl chain exhibit a higher order parameter value than the series member with the even carbon number (Fig. 5). The same alternation is also observed for the nematic-isotropic transition temperature, $T_{\mathrm{NI}}$ (see Table I).

The agreement of the order parameter values determined by using various experimental methods is quite good; the differences do not exceed $10 \%$. Previously many authors $[5,27,28]$ had observed the difference between the order parameter values obtained from various methods. It is rather difficult to estimate the absolute scale of $S$ with the same accuracy. The essential advantage of the methods applied here by us is their simplicity and although the results obtained are perhaps not the absolute values of order parameter, they allow us to ascertain and compare 


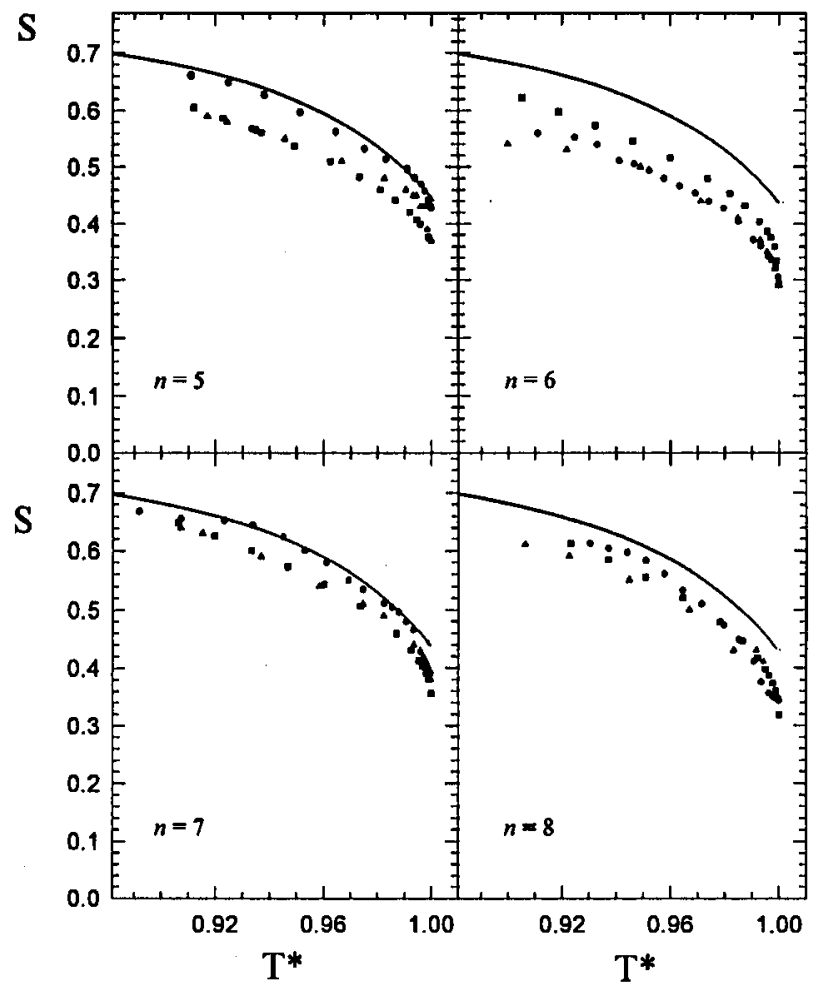

Fig. 4. Temperature dependence of order parameter $S$ for liquid crystals studied determined from optical birefringence (circles), dielectric anisotropy (squares) and polarized absorption spectra of DANS (triangles). Solid line - Maier-Saupe theory.

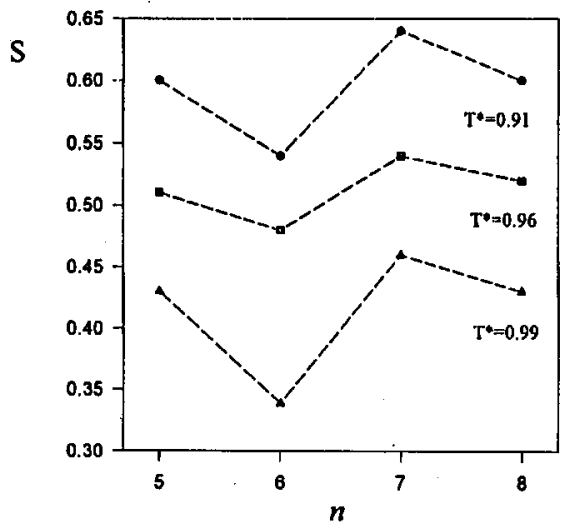

Fig. 5. Odd-even effect in order parameter $S$ for homologous series of bicyclo[2,2,2]octane derivatives with - NCS end group. 
TABLE II

Order parameter $S$ for nematics doped with DANS.

\begin{tabular}{c|c|c|c}
\hline \hline $\begin{array}{c}\text { Liquid } \\
\text { crystal }\end{array}$ & Molecular structure & $\begin{array}{c}S \\
T^{*}=0.97\end{array}$ & $\begin{array}{c}S \\
T^{*}=0.99\end{array}$ \\
\hline 1 & $\mathrm{C}_{6} \mathrm{H}_{13}-\square-20-\mathrm{NCS}$ & 0.44 & 0.40 \\
2 & $\mathrm{C}_{6} \mathrm{H}_{13}-\square-\square-\mathrm{NCS}$ & $0.54^{a}$ & $0.44^{a}$ \\
3 & $\mathrm{C}_{6} \mathrm{H}_{13}-\square-\square-\mathrm{CN}$ & $0.42^{a}$ & $0.35^{a}$ \\
4 & $\mathrm{C}_{6} \mathrm{H}_{13}-\square \mathrm{O}-0 \mathrm{CN}$ & $0.45^{a}$ & $0.40^{a}$ \\
\hline
\end{tabular}

${ }^{a}$ from Ref. [29]

the orienting ability of various liquid crystals investigated, which is very important information from the technological point of view.

Finally, let us compare the values of the order parameter for the compounds investigated in this paper with $S$ data for the homologues of other liquid crystals series. To make this, in Table II the order parameter values, $S$, obtained from the polarized absorption measurements of DANS dissolved in one of the members $(n=6)$ of various liquid crystalline homologous series at two reduced temperatures are gathered. It is seen that the order parameter of the liquid crystal with terminal -NCS group is higher than that for analogous compound with -CN group and that the $S$ value decreases as the ring at the alkyl group is changed from benzene through cyclohexane to bicyclooctane.

\section{Acknowledgments}

This paper was partially supported by the project No. 2 P302 27503 of the State Committee for Scientific Research (Republic of Poland). D.B. and A.W. thank Poznań University of Technology for financial support.

\section{References}

[1] W. Maier, A. Saupe, Z. Nat.forsch. A 14, 882 (1959); 15, 287 (1960).

[2] R.L. Humphries, P.G. James, G.R. Luckhurst, J. Chem. Soc. Faraday Trans. 68, 1031 (1972).

[3] R. Dąbrowski, Mol. Cryst. Liq. Cryst. 191, 17 (1990).

[4] R. Dąbrowski, J. Dziaduszek, W. Drzewiński, K. Czupryński, Z. Stolarz, Mol. Cryst. Liq. Cryst. 191, 171 (1990). 
[5] B. Żywucki, W. Kuczyriski, G. Czechowski, SPIE, in press.

[6] A. Saupe, Z. Nat.forsch. A 19, 161 (1964).

[7] P.G. de Gennes, The Physics of Liquid Crystals, Clarendon Press, Oxford 1974.

[8] V. Zvetkoff, Acta Physicochim. URSS 16, 132 (1942).

[9] G. Vertogen, W.H. de Jeu, Thermotropic Liquid Crystals, Fundamentals, Springer Verlag, Berlin 1988.

[10] I. Haller, Prog. Solid State Chem. 10, 103 (1975).

[11] A. Buka, W.H. de Jeu, J. Phys. (Paris) 43, 361 (1982).

[12] G.H. Heilmeier, L.A. Zanoni, Appl. Phys. Lett. 13, 91 (1968).

[13] G.H. Heilmeier, J.A. Castellano, L.A. Zanoni, Mol. Cryst. Liq. Cryst. 8, 293 (1969).

[14] R.W. Filas, M.M. Labes, J. Appl. Phys. 52, 3949 (1981).

[15] A.V. Ivashchenko, O.S. Petrova, V.V. Titov, Mol. Cryst. Liq. Cryst. 108, 5 (1984).

[16] D.L. White, G.N. Taylor, J. Appl. Phys, 45, 4718 (1974).

[17] D. Bauman, Mol. Cryst. Liq. Cryst. 159, 197 (1988).

[18] K.J. Mainusch, U. Müller, P. Pollmann, H. Stegemeyer, Z. Nat.forsch. A 27, 1677 (1962).

[19] T. Hanemann, private communication.

[20] J. Jadżyn, G. Czechowski, N.T. Shonova, Liq. Cryst. 3, 1637 (1988).

[21] B.R. Ratna, R. Shashidar, Mol. Cryst. Liq. Cryst. 42, 113 (1977).

[22] S. Sen, K. Kali, S.K. Roy, S.B. Roy, Mol. Cryst. Liq. Cryst. 126, 269 (1985).

[23] N.V. Madhusudana, S. Chandrasekhar, Pramana (Suppl.) 1, 57 (1975).

[24] A.J. Leadbetter, R.M. Richardson, C.N. Colling, J. Phys. (Paris) 36, C1 (1975).

[25] H. Kelker, R. Hatz, Handbook of Liquid Crystals, Verlag Chemie, Weinheim 1980, p. 48 .

[26] S. Marcelja, J. Chem. Phys. 60, 3599 (1974).

[27] A.J. Leadbetter, in: The Molecular Physics of Liquid Crystals, Eds. G.H. Luckhurst, G.W. Gray, Academic Press, London 1979, p. 285.

[28] D. Bauman, habilitation dissertation, Poznan 1990.

[29] H. Moryson, D. Bauman, J. Jadżyn, Mol. Cryst. Liq. Cryst. 250, 63 (1994). 